\title{
ESTUDO COMPARATIVO ENTRE MARCADORES ULTRA-SONOGRÁFICOS MORFOLÓGICOS PREDITORES DE PARTO PRÉ-TERMO: SINAL DO AFUNILAMENTO DO COLO E AUSÊNCIA DO ECO GLANDULAR ENDOCERVICAL*
}

\author{
Claudio Rodrigues Pires ${ }^{1}$, Antonio Fernandes Moron ${ }^{2}$, Rosiane Mattar ${ }^{3}$, Luiz Kulay Júnior ${ }^{4}$
}

Resumo OBJETIVO: Avaliar o risco de parto pré-termo (PPT) espontâneo na população geral a partir do estudo comparativo dos marcadores ultra-sonográficos morfológicos do colo uterino, como o sinal do afunilamento e a ausência da área glandular endocervical. MATERIAIS E MÉTODOS: Foram arroladas 361 gestantes na popu-

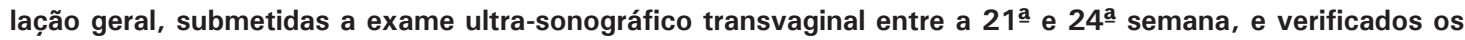
resultados perinatais. RESULTADOS: $A$ incidência de PPT espontâneo foi de 5,0\%. 0 sinal do afunilamento foi observado em $4,2 \%$ da população estudada e em $22,2 \%$ das pacientes que evoluíram para PPT espontâneo. Tal parâmetro mostrou associação significante com PPT (p $<0,001$; risco relativo de 6,68). A ausência do eco glandular endocervical (EGE) foi detectada em 2,8\% das pacientes estudadas e em 44,4\% das pacientes que evoluíram para PPT espontâneo. Este parâmetro demonstrou forte associação com PPT espontâneo ( $p<0,001$; risco relativo de 28,57). A análise de regressão logística multivariada apontou a ausência do EGE como a única variável morfológica associada ao PPT espontâneo. CONCLUSÃO: A predição do PPT espontâneo a partir de sinais ultra-sonográficos deve ser realizada contemplando marcadores biométricos e morfológicos, entre estes, a ausência do EGE. Este estudo indica uma tendência clara da marcante importância da ausência do EGE como indicador do risco para PPT espontâneo, a ser confirmada futuramente em pesquisas multicêntricas.

Unitermos: Colo uterino; Gravidez; Parto pré-termo; Ultra-sonografia transvaginal.

Abstract A comparative study between morphologic ultrasonographic markers for preterm delivery: funneling sign and absence of cervical gland area.

OBJECTIVE: To verify if different morphological ultrasonographic markers such as the funneling sign and the cervical gland area can be predictors of spontaneous premature delivery in pregnant women between 21 and 24 weeks of gestation. MATERIALS AND METHODS: This was a prospective cross-sectional study in which 361 women with 21 to 24 weeks of gestation were examined by transvaginal ultrasonography. The gestational age at delivery was later checked by telephone or mail. RESULTS: Spontaneous preterm delivery (SPD) occurred in $5 \%$ of the patients. Cervical funneling occurred in $4.2 \%$ of the women and in $22.2 \%$ of those who had SPD. This finding was correlated to preterm delivery $(p<0.001$; relative risk of 6.68). Absence of a hypoechoic area peripheral to the cervical canal consistent with endocervical epithelium glands, namely endocervical glandular echo (EGE) feature, was detected in $2.8 \%$ of all patients and in $44.4 \%$ of those who developed spontaneous preterm labor. There was a statistically significant association of this feature to SPD ( $p<0.001$; relative risk of 28.57). Multivariance logistic regression analysis showed that this was the feature with strongest correlation with SPD, when compared to cervical funneling. CONCLUSION: Prediction of SPD through ultrasound features should observe biometrical and morphological signs such as absence of EGE. This is a new and important ultrasound finding that can be considered a predictor of risk for SPD, and should be confirmed in future multicentric trials.

Key words: Cervical gland area; Pregnancy; Preterm delivery; Transvaginal ultrasonography.

\footnotetext{
* Trabalho realizado na Universidade Federal de São Paulo/ Escola Paulista de Medicina (Unifesp/EPM), São Paulo, SP.

1. Doutor em Medicina pela Unifesp/EPM, Membro Titular do Colégio Brasileiro de Radiologia e Diagnóstico por Imagem (CBR)

2. Livre-Docente, Coordenador do Curso de Pós-Gradução de Obstetrícia da Unifesp/EPM.

3. Professora Adjunta do Departamento de Obstetrícia da Unifesp/EPM.

4. Professor Titular, Chefe do Departamento de Obstetrícia da Unifesp/EPM.

Endereço para correspondência: Dr. Claudio Rodrigues Pires. Rua Capitão Garcindo, 145, Pacaembu. São Paulo, SP, 01250 010. E-mail: crpires@uol.com.br

Recebido para publicação em 17/3/2004. Aceito, após revisão, em 14/4/2004.
}

\section{INTRODUÇÃO}

A prematuridade tem sido identificada, nos dias atuais, como um dos principais problemas de saúde no período perinatal, constituindo a causa mais importante de morbidade e de mortalidade neonatal ${ }^{(1-4)}$. O parto pré-termo (PPT), ou seja, o que ocorre antes da $37^{\mathrm{a}}$ semana completa, constitui importante determinante de mor- bidade neonatal e infantil, incluindo alterações do desenvolvimento neuromotor, doenças respiratórias crônicas, predisposição para processos infecciosos e distúrbios oftalmológicos ${ }^{(5)}$. Embora existam muitas características maternas e fetais associadas ao PPT, a sua etiologia frequientemente não pode ser definida ${ }^{(4,6)}$, e as causas idiopáticas representam cerca de $20 \%$ a $40 \%$ dos $\operatorname{casos}^{(7,8)}$. 
Muitas publicações destacaram métodos clínicos, marcadores biológicos, bioquímicos e ultra-sonográficos, que, aplicados isolada ou combinadamente, buscam predizer o PPT espontâneo ${ }^{(\mathbf{9 - 1 2})}$. A prevenção do PPT espontâneo, entretanto, permanece um grande desafio para os profissionais da saúde ${ }^{(\mathbf{1 , 6})}$.

O exame por ultra-sonografia transvaginal (US-TV) consiste no método mais apropriado para o estudo do colo uterino durante a gravidez, pois permite avaliar a forma e a biometria cervical com alto grau de confiabilidade ${ }^{(13)}$. Há mais de uma década, acumulam-se trabalhos estabelecendo as medidas cervicais preditoras do PPT, sendo o comprimento cervical a principal variável utilizada. A maioria dessas publicações apresenta uma conclusão comum: a de que, quanto menor o comprimento cervical, maior o risco para PPT espontâneo ${ }^{(\mathbf{1 4 - 2 1 )}}$. Dessa forma, embora diferindo muito no que se refere aos métodos e populações utilizados, os estudos foram estabelecendo medidas de referência que podem ser utilizadas como preditoras ${ }^{(\mathbf{1 3})}$. O limite do comprimento do colo uterino representativo de risco para PPT espontâneo ainda é motivo de controvérsia na literatura, e diversos estudos têm revelado como pontos de corte comprimentos que variam

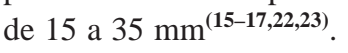

Permanece controverso o significado das modificações morfológicas da cérvice uterina durante a gravidez evidenciadas à US. Uma das características do canal cervical visualizada por US utilizada na predição do risco de prematuridade é o sinal do afunilamento ou dilatação do orifício interno do colo $^{(\mathbf{2 3 - 2 5})}$. O processo de dilatação cervical inicia-se pela abertura do orifício interno, o qual pode ser evidenciado à US como imagem anecóica em formato de funil na porção superior do canal endocervical, com protrusão das membranas para o interior do canal cervical. Esta medida é especialmente interessante, pois a dilatação do orifício interno freqüentemente está presente sem a dilatação do orifício externo ${ }^{(24)} \mathrm{e}$, portanto, antecederia o diagnóstico clínico pelo toque vaginal.

Diversas publicações destacam as mudanças que ocorrem durante a realização do exame, com o aparecimento do sinal do afunilamento intermitente e a conseqüente redução do comprimento cervical. Por causa desses eventos, muitos investigadores têm recomendado prolongar a observação por pelo menos três minutos ${ }^{(25-27)}$.

O sinal do afunilamento ou dilatação do orifício interno do colo é achado ecográfico morfológico durante a gestação, descrito como indicativo de risco para insuficiência istmo-cervical e PPT por diversos

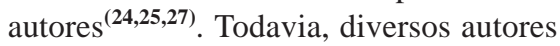
não reconheceram o sinal do afunilamento como preditor de PPT. Em tese elaborada na Universidade Federal de São Paulo/Escola Paulista de Medicina (Unifesp/ EPM), a presença do sinal do afunilamento em gestantes com antecedentes de PPT não apresenta correlação com recorrência de prematuridade ${ }^{(\mathbf{2 8})}$. Em estudo realizado no King's College Hospital de Londres, To et al. avaliaram, por meio da US-TV, 6.334 pacientes com gestação única entre a $22^{a}$ e 24 ${ }^{\mathrm{a}}$ semana. O sinal do afunilamento do orifício interno, considerado quando sua largura excedia $5 \mathrm{~mm}$, esteve presente em cerca de $4 \%$ das gestações e a prevalência do sinal diminuiu com o aumento do comprimento do colo. A análise de regressão logística demonstrou que o sinal do afunilamento não contribui significativamente para aumentar a predição do PPT em idade gestacional anterior a 33 semanas $^{(\mathbf{2 1})}$. Em 2001, Owen et al. indicaram que o afunilamento do orifício interno apresenta ampla variação biométrica populacional, fato este que pode limitar a reprodutibilidade deste achado ultra-sonográfico enquanto o comprimento cervical é reprodutível e clinicamente útil ${ }^{(27)}$. A conclusões semelhantes chegaram Iams et al., os quais assinalaram variabilidade interobservador importante ${ }^{(\mathbf{1 5})}$. Hassan et al. descreveram que a dificuldade na obtenção de uma medida reprodutível do sinal do afunilamento tem induzido muitos pesquisadores a usar a medida do comprimento cervical como o método de eleição no rastreamento do $\mathrm{PPT}^{(\mathbf{1 9 )}}$.

A não-visualização ecográfica da região correspondente ao epitélio endocervical consiste em outro sinal ultra-sonográfico morfológico descrito recentemente que poderia ser considerado preditor de prematuridade. Os aspectos histológicos da ectocérvice, endocérvice e estroma cervical podem ser observados na Figura 1. Porém, apenas três publicações destacaram este marcador ecográfico ${ }^{(29-31)}$.

Em Tóquio, no final da década passada, pesquisadores da Nippon Medical School, liderados por Sekiya, dedicaramse à observação da maturação do colo uterino em gestantes por meio da visualização da região correspondente ao epitélio endocervical por US-TV. Os autores realizaram estudo longitudinal em 260 pacientes com gestações únicas durante o período de 16 a 41 semanas, comparando os dados do exame de toque vaginal com achados ultra-sonográficos morfológicos das glândulas endocervicais. Nomearam de "cervical gland area" à faixa habitualmente hipoecóica ou, mais raramente, hiperecóica, observada na projeção das glândulas endocervicais ${ }^{(29)}$. A detecção da imagem correspondente à área glandular cervical foi possível, nesse trabalho, em $100 \%$ dos casos até a 27ª semana de gestação, com significativa redução nas semanas seguintes.

Em publicação do mesmo grupo no ano de 2002, foram analisadas 101 pacientes com trabalho de PPT em tratamento clínico e 260 gestantes controles, todas avaliadas por meio de toque vaginal e US-TV para mensuração do comprimento cervical e detecção da área glandular cervical. Foi observada importante correlação entre as taxas de detecção das glândulas endocervicais e comprimento cervical, índice de maturação cervical e resultado da gestação. Segundo o estudo, a ausência da visualização da área glandular cervical também indica maturação cervical e pode ser considerada preditora de maus resultados nas pacientes com trabalho de $\mathrm{PPT}^{(30)}$.

No ano seguinte, Fukami et al., do mesmo grupo, estudaram a área glandular cervical em pacientes entre 16 e 19 semanas de gestação, por meio da US-TV. Os autores observaram 96 pacientes que evoluíram para PPT espontâneo, porém apenas oito em idade gestacional inferior à $32^{\mathrm{a}}$ semana. Na ocasião, compararam a ausência de visualização da área glandular cervical com o comprimento do canal endocervical e observaram maior sensibilidade (sensibilidade de $75 \%$ versus sensibilidade de $50 \%$ ) e uma significativa elevação do valor preditivo positivo (sensibilidade de $54,5 \%$ versus sensibilidade de $8,3 \%$ ) para PPT anterior à 32 a semana de gestação. Os 
autores concluem que a ausência da visualização da área glandular cervical constitui novo e útil parâmetro ultra-sonográfico na predição de PPT espontâneo ${ }^{(31)}$.

Assim, tivemos a intenção de avaliar se o estudo morfológico e biométrico do colo uterino, por ocasião do exame ultra-sonográfico obstétrico morfológico fetal do segundo trimestre, auxiliaria na predição do PPT, o qual representa grande preocupação de radiologistas, obstetras e neonatologistas. Este estudo teve por objetivos verificar a prevalência de dois marcadores morfológicos - o sinal do afunilamento cervical e a ausência da "área das glândulas endocervicais" — na mesma população e na mesma idade gestacional e suas relações com PPT espontâneo. Procurou também avaliar a relação entre os parâmetros morfológicos entre si, buscando estabelecer o impacto relativo na predição do PPT espontâneo.

\section{MATERIAIS E MÉTODOS}

O presente estudo foi analisado e aprovado pelo Comitê de Ética em Pesquisa da Unifesp/EPM-Hospital São Paulo. Foi desenvolvido de forma prospectiva, transversal e incluiu uma população de mulheres grávidas submetidas a exame ultra-sonográfico obstétrico de rotina no primeiro trimestre encaminhadas no período de outubro de 1999 a outubro de 2001. Inicialmente, participaram desta pesquisa 685 gestantes, as quais apresentavam feto único e vivo por ocasião do exame ecográfico do primeiro trimestre e concordaram em participar do estudo.

A avaliação ecográfica obstétrica por via abdominal até a $14^{\underline{a}}$ semana permitiu a identificação da idade gestacional de maneira acurada, a qual foi utilizada como parâmetro básico para estimativa da idade da gravidez por ocasião do exame ultrasonográfico morfológico do segundo trimestre e no dia do parto. As mesmas pacientes foram convocadas a retornar no período da $21^{\mathrm{a}}$ à $24^{\mathrm{a}}$ semanas gestacionais. Compareceram no período marcado e obedeceram aos critérios de inclusão 425 pacientes, as quais foram submetidas a uma segunda avaliação ecográfica morfológica fetal, efetuada por via abdominal, seguida do estudo por US-TV do colo uterino.
Os critérios de exclusão do estudo foram: malformações müllerianas, gestações múltiplas, malformações fetais, óbito fetal, alterações da quantidade de líquido amniótico, placenta com inserção segmentar, antecedentes de cirurgia no colo uterino (conização, amputação, cerclagem) e procedimentos cirúrgicos durante a gestação. Quarenta e duas mulheres foram retiradas da amostra por apresentarem algum critério de exclusão ou por perda de acompanhamento pós-exame e, portanto, desconhecimento dos dados do resultado gestacional. Também foram excluídas as mulheres submetidas a indução de parto antes do término da gestação, por diferentes razões. Foram identificadas 40 pacientes com PPT, sendo 22 eletivos e 18 espontâneos. As causas que motivaram os PPT induzidos foram: hipertensão arterial crônica e/ou pré-eclâmpsia em sete pacientes, óbito fetal em duas, malformação fetal (megabexiga-microcólon) em uma e polidrâmnio grave em uma. Nove outras mulheres tiveram suas gestações resolvidas por meio de parto cesáreo e duas pela indução do parto por via vaginal sem indicações declaradas. Dessa forma, a amostragem final totalizou 361 gestantes.

Apenas um médico realizou todas as medidas e avaliações ultra-sonográficas morfológicas cervicais, obedecendo rigorosamente aos passos discutidos a seguir.

A avaliação por US-TV foi efetivada com diferentes equipamentos, de alta resolução, com transdutores endocavitários multifreqüenciais e ângulo de visão igual ou superior a $90^{\circ}$, devidamente calibrados para mensurações e apropriados para tal procedimento.

Antes dos exames de US-TV, as pacientes foram orientadas para proceder ao esvaziamento vesical completo. Posteriormente, foram colocadas em posição de litotomia, em decúbito dorsal, com os membros inferiores abduzidos, dispostos de forma simétrica, permitindo a livre movimentação do transdutor. $\mathrm{O}$ transdutor foi, então, posicionado no terço médio da vagina para obtenção dos cortes sem exercer compressão sobre a cérvice, de maneira a não distorcer a imagem e, desta forma, não provocar falso alongamento do colo ou desaparecimento do sinal do afunilamento, quando presente.
Após a identificação do orifício interno do colo, o transdutor foi movimentado até a obtenção da imagem de toda a extensão do canal cervical, usualmente rodeado por uma faixa hipoecogênica ou hiperecogênica identificada como "área das glândulas endocervicais"(29-31). Uma imagem adequada foi definida como a visualização nítida do orifício interno do colo, do orifício externo e de toda a extensão do canal endocervical. $\mathrm{O}$ estroma do colo uterino mostra-se, em condições habituais, moderadamente ecogênico e homogêneo à US. O campo visual foi ampliado, de tal forma que a imagem do colo uterino ocupasse cerca de três quatros da tela do aparelho de US.

A "área das glândulas endocervicais", mencionada por Sekiya et al. e outros autores, foi denominada, neste trabalho, de "eco glandular endocervical" (EGE) e definida como uma zona hipoecóica ou hiperecóica em torno do canal endocervical (Figuras 2 e 3), a qual corresponde à área histológica do epitélio glandular endocervical (29-31) (Figura 1). Quando não visualizado, o EGE em torno do canal endocervical era definido como ausente (Figura 4). $\mathrm{O}$ sinal do afunilamento foi considerado presente quando a abertura do orifício interno apresentava forma de "U"ou "V" cuja largura excedia 5 mm (Figura 4).

Após a realização do exame entre a $21^{\text {a }}$ e a 24ํㅗ semana, as gestantes prosseguiram o acompanhamento pré-natal, segundo as rotinas do serviço onde o realizavam, sem interferência do pesquisador na conduta obstétrica adotada. Este estudo não teve como objetivo o acompanhamento clínico pré-natal das pacientes envolvidas na pesquisa. Os dados relacionados à data do parto, via de parto e condições do recémnascido foram obtidos por meio de telefonemas para cada uma das gestantes, aproximadamente 20 semanas após a realização do exame por US. As pacientes não localizadas por esta via foram contatadas através de carta explicativa, com um questionário de fácil compreensão. As informações coletadas por via telefônica ou por meio da ficha de resposta postada foram transferidas para a ficha cadastral, da qual foram então transcritas para um sistema de análise de dados em computador com planilha eletrônica Microsoft Access ${ }^{\circledR}$. 


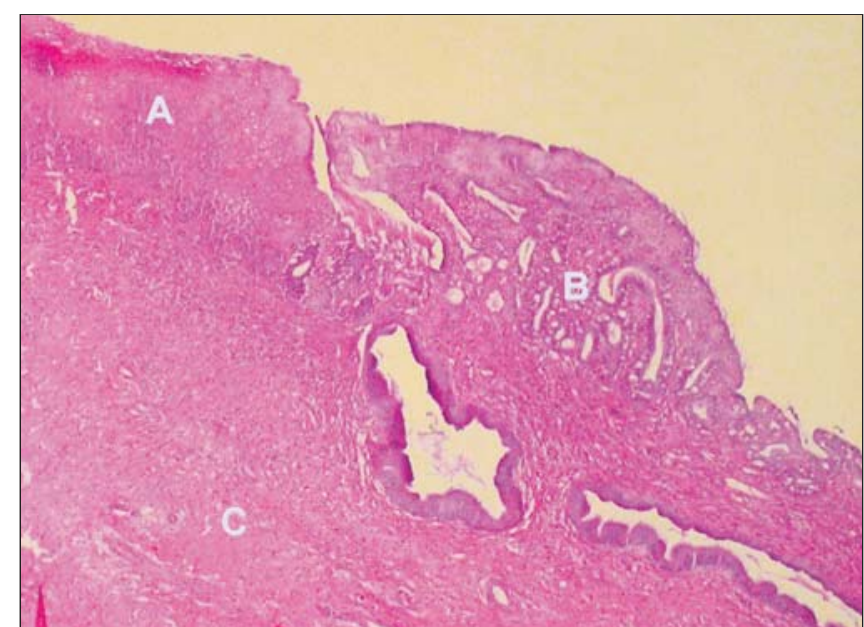

Figura 1. Fotomicrografia do colo uterino corado pelo método hematoxilinaeosina. Observa-se a ectocérvice constituída por epitélio escamoso pavimentado (A), glândulas endocenvicais (B) e estroma cenvical (C). Fotografia gentilmente cedida pela patologista Ângela Logullo (Unifesp/EPM).

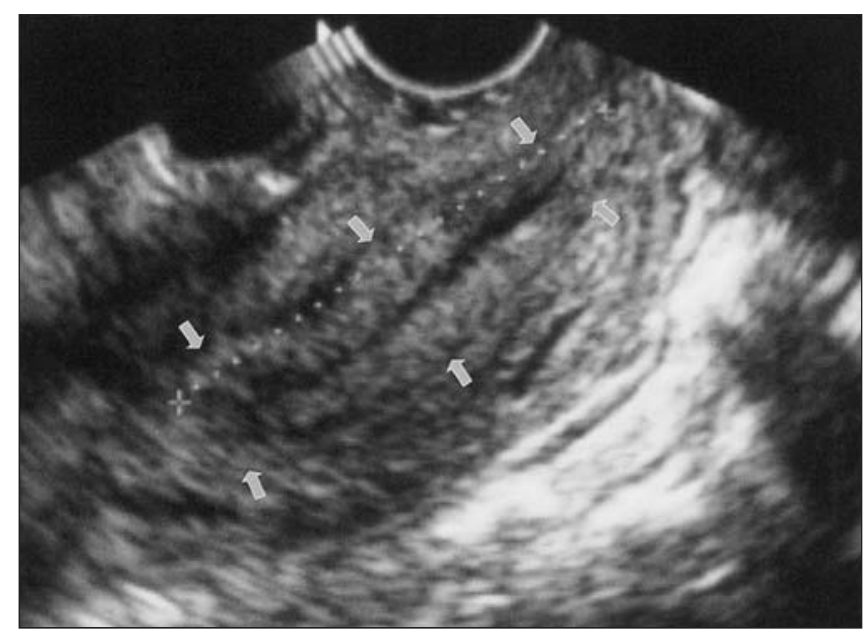

Figura 3. Exame ultra-sonográfico que revela colo uterino em corte longitudinal. As setas apontam a transição entre a área das glândulas endocervicais (aspecto hiperecóico) e o estroma cervical periférico.

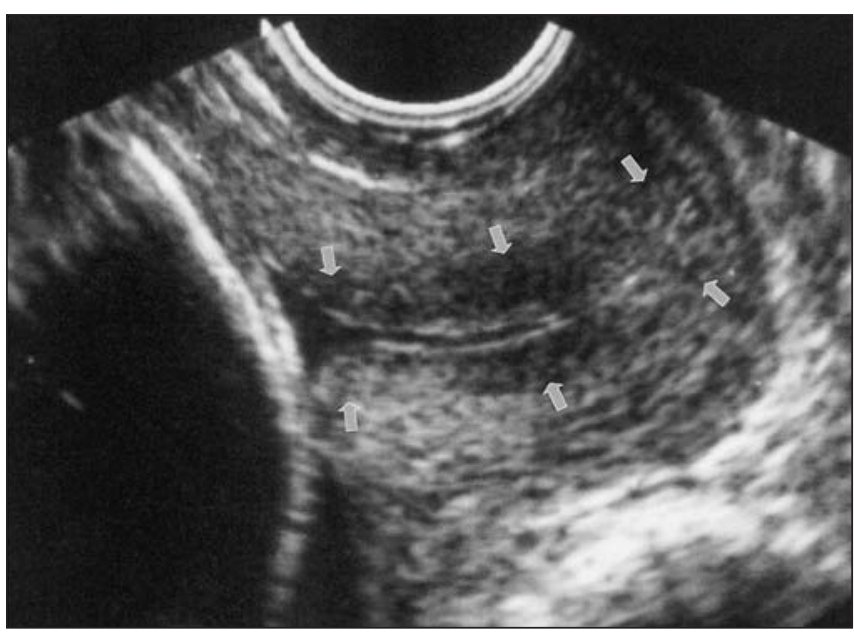

Figura 2. Exame ultra-sonográfico que evidencia colo uterino em corte longitudinal. As setas apontam a transição entre a área das glândulas endocenvicais (aspecto hipoecóico) e o estroma cenvical. Obsenva-se o canal endocenvical com pequena quantidade de muco (anecóico).

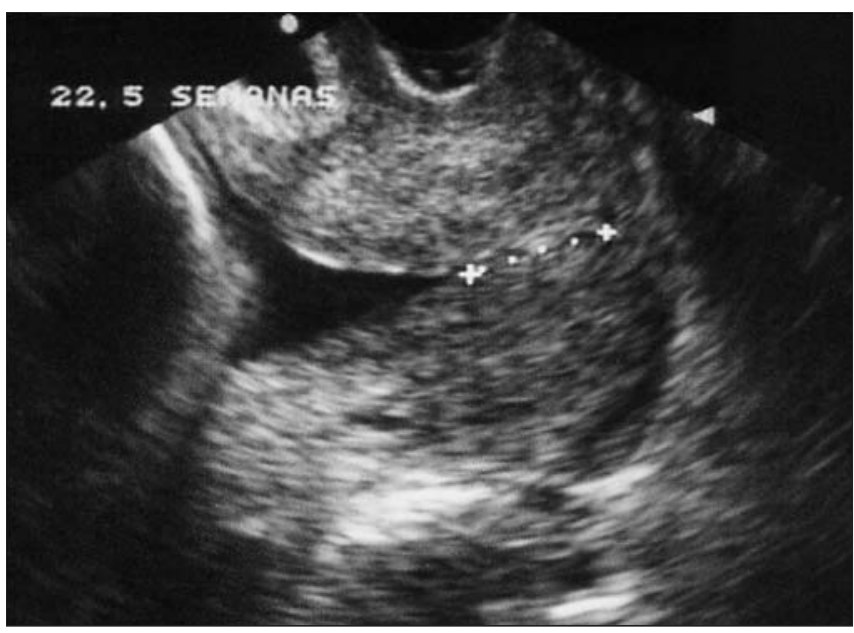

Figura 4. Exame ultra-sonográfico que mostra colo uterino em corte longitudinal. Observa-se o sinal do "afunilamento" e comprimento residual reduzido. Não foi, neste exemplo, evidenciado o "eco glandular endocervical".
As variáveis qualitativas foram representadas por frequiência absoluta e relativa, e as variáveis quantitativas, por média, desvio-padrão, mediana e valores mínimo e máximo. Todos os dados coletados foram descritos para caracterizar a amostra estudada. O nível de significância adotado foi de $95 \%($ alfa $=5 \%$ ) e descritivos ( $\mathrm{p}$ ) iguais ou inferiores a 0,05 foram considerados significantes. A associação entre variáveis qualitativas foi avaliada pelo teste do quiquadrado ou teste exato de Fisher. Para cada variável estudada, foi calculado o risco relativo seguido do intervalo com $95 \%$ de confiança (IC). A técnica de análise de regressão logística univariada e multiva- riada foi utilizada para selecionar, dentre as variáveis estudadas, quais foram preditivas para o PPT espontâneo e qual o grau dessa relação ("odds ratio"), segundo Hosmer e Lemesshow ${ }^{(32)}$.

\section{RESULTADOS}

Os dados mais relevantes encontrados nesta pesquisa são apresentados a seguir, na forma de tabelas, dispostas de modo seqüencial de acordo com avaliação morfológica isolada e associativa.

No dia da realização do exame ultra-sonográfico, a distribuição da idade gestacional corrigida das gestantes foi: oito pacien- tes $(2,2 \%)$ com 21 semanas, $61(16,9 \%)$ com 22 semanas, $194(53,7 \%)$ com 23 semanas e $98(27,1 \%)$ com 24 semanas. Das 361 gestantes avaliadas, $18(5,0 \%)$ apresentaram PPT espontâneo em idades gestacionais variando entre 27 e 36 semanas, sendo $11(3,0 \%)$ em idade gestacional inferior a 36 semanas, oito $(2,2 \%)$ em idade gestacional inferior a 35 semanas, cinco $(1,4 \%)$ em idade gestacional inferior a 34 semanas e duas pacientes $(0,6 \%)$ em idade gestacional inferior a 33 semanas. Quanto ao tipo de parto, 159 pacientes $(44,0 \%)$ informaram parto cesáreo, 88 (24,4\%) partos normais espontâneos e 114 (31,5\%) partos normais induzidos. 
O sinal do afunilamento foi observado em $15(4,2 \%)$ do total de 361 pacientes e em quatro $(22,2 \%)$ das 18 pacientes que evoluíram para PPT espontâneo. Os valores de sensibilidade, especificidade, valor preditivo positivo (VPP) e valor preditivo negativo (VPN) do sinal ultra-sonográfico morfológico, de acordo com as idades gestacionais dos PPT, encontram-se descritos na Tabela 1. A análise univariada do sinal do afunilamento evidenciou $\mathrm{p}<0,001$ na predição de PPT.

Na amostra de 361 gestantes, foi observada ausência do sinal EGE em dez (2,8\%) pacientes. Dentre as 18 mulheres que tiveram PPT espontâneo, o EGE não foi observado em oito, ou seja, em $44,4 \%$ das gestantes. A sensibilidade, especificidade, VPP e VPN da ausência do EGE associa- da ao PPT observados nas diferentes idades gestacionais encontram-se demonstrados na Tabela 2. A análise univariada da ausência do EGE evidenciou p < 0,001 na predição de PPT.

Detectou-se também associação estatisticamente significante entre os parâmetros ultra-sonográficos morfológicos estudados e a ocorrência de PPT espontâneo ( $\mathrm{p}<$ $0,05)$, indicando que a presença de alteração nesses parâmetros é significantemente maior no grupo de pacientes que evolui para PPT quando comparado ao grupo de mulheres com parto no termo (Tabela 3).

Diante dos resultados que apontaram associação entre os marcadores morfológicos e o PPT espontâneo, pesquisamos de forma comparativa o poder das diferentes variáveis. O resultado da análise de regres-

Tabela 1 Presença do sinal do afunilamento e associação com parto pré-termo espontâneo.

\begin{tabular}{|l|c|c|c|c|c|c|}
\hline \multirow{2}{*}{$\begin{array}{l}\text { Sinal do } \\
\text { afunilamento }\end{array}$} & \multicolumn{6}{|c|}{ Idade gestacional ao nascimento } \\
\cline { 2 - 7 } presente & \multicolumn{2}{|c|}{$<37$ semanas } & \multicolumn{2}{c|}{$<36$ semanas } & \multicolumn{2}{c|}{$<35$ semanas } \\
\cline { 2 - 7 } & $\mathrm{N}$ & $\%$ & $\mathrm{~N}$ & $\%$ & $\mathrm{~N}$ & $\%$ \\
\hline Sensibilidade & $4 / 18$ & 22,2 & $4 / 11$ & 36,4 & $4 / 8$ & 50,0 \\
Especificidade & $332 / 343$ & 96,8 & $339 / 350$ & 96,9 & $342 / 353$ & 96,9 \\
VPP & $4 / 15$ & 26,7 & $4 / 15$ & 26,7 & $4 / 15$ & 26,7 \\
VPN & $332 / 346$ & 96,0 & $339 / 346$ & 98,0 & $342 / 346$ & 98,8 \\
\hline
\end{tabular}

VPP, valor preditivo positivo; VPN, valor preditivo negativo.

Tabela 2 Ausência de eco glandular endocervical e associação com parto pré-termo espontâneo.

\begin{tabular}{|l|c|c|c|c|c|c|}
\hline \multirow{2}{*}{$\begin{array}{l}\text { Eco glandular } \\
\text { endocervical } \\
\text { ausente }\end{array}$} & \multicolumn{6}{|c|}{ Idade gestacional ao nascimento } \\
\cline { 2 - 7 } & $\mathrm{N}$ & $\%$ & $\mathrm{~N}$ semanas & $\% 36$ semanas & \multicolumn{2}{c|}{$<35$ semanas } \\
\cline { 2 - 7 } Sensibilidade & $8 / 18$ & 44,4 & $7 / 11$ & 63,6 & $6 / 8$ & 75,0 \\
Especificidade & $341 / 343$ & 99,4 & $347 / 350$ & 99,1 & $349 / 353$ & 98,9 \\
VPP & $8 / 10$ & 80,0 & $7 / 10$ & 70,0 & $6 / 10$ & 60,0 \\
VPN & $341 / 351$ & 97,2 & $347 / 351$ & 98,9 & $349 / 351$ & 99,4 \\
\hline
\end{tabular}

VPP, valor preditivo positivo; VPN, valor preditivo negativo.

são logística multivariada revela que a ausência do EGE mostrou impacto associativo significantemente maior com o PPT espontâneo anterior a 37 semanas $(\mathrm{p}<0,001$; "odds ratio" $=70,198 ; \mathrm{IC}_{95 \%}=11,21 \mathrm{a}$ 439,52) quando comparado ao sinal do afunilamento $(\mathrm{p}=0,758$; "odds ratio" $=$ 0,$704 ; \mathrm{IC}_{95 \%}=0,08$ a 6,59). O mesmo resultado foi encontrado quando estudados os casos de PPT que ocorreram em período anterior a 36 semanas, 35 semanas (EGE ausente com p < 0,001, "odds ratio" $=57,482, \mathrm{IC}_{95 \%}=6,62$ a 498,92 ; sinal do afunilamento com $\mathrm{p}=0,598$, "odds ratio" $=1,931, \mathrm{IC}_{95 \%}=0,17$ a 22,25$)$ e 34 semanas. Essa análise não foi aplicada para prematuridade em idades gestacionais anteriores à $33^{\underline{a}}$ semana, em decorrência do pequeno número de casos nesta condição.

\section{DISCUSSÃO}

Foram estudadas pacientes da população geral, ressaltando que poucas são as publicações que abordaram este tipo de amostragem na literatura médica nacional, sem qualquer tipo de triagem prévia das gestantes, relacionada a algum risco conhecido para PPT, tal como antecedentes obstétricos. Como pudemos observar pela distribuição das idades gestacionais no dia do parto, ocorreram 18 PPT espontâneos na população de 361 pacientes, ou seja, 5,0\% das pacientes estudadas. Se considerarmos também os PPT eletivos, obtivemos o índice total de PPT de 8,3\%. Este índice é muito semelhante aos dados existentes na literatura nacional sobre $\mathrm{PPT}^{(33,34)}$.

Decidimos avaliar pacientes com um estudo transversal no período de 21 a 24 semanas, pois não há consenso sobre a necessidade do exame ultra-sonográfico do

Tabela 3 Distribuição dos casos com sinal do afunilamento e dos casos de ausência do eco glandular endocervical na amostra total e nas gestantes que tiveram parto pré-termo espontâneo e a termo.

\begin{tabular}{|c|c|c|c|c|c|c|c|c|c|}
\hline \multirow[b]{2}{*}{ Parâmetro ultra-sonográfico } & & \multicolumn{2}{|c|}{$\begin{array}{l}\text { Amostra total } \\
\qquad(n=361)\end{array}$} & \multicolumn{2}{|c|}{$\begin{array}{l}\text { Idade gestacional } \\
\text { ao nascimento } \\
<37 \text { semanas } \\
\quad(n=18)\end{array}$} & \multicolumn{2}{|c|}{$\begin{array}{l}\text { Idade gestacional } \\
\text { ao nascimento } \\
\begin{array}{c}\geq 37 \text { semanas } \\
(n=343)\end{array}\end{array}$} & \multirow[b]{2}{*}{$p$} & \multirow{2}{*}{$\begin{array}{l}\text { Risco relativo } \\
{\left[\mathrm{IC}_{95 \%}\right]}\end{array}$} \\
\hline & & $\mathrm{N}$ & $\%$ & $\mathrm{~N}$ & $\%$ & $\mathrm{~N}$ & $\%$ & & \\
\hline Sinal do afunilamento & $\begin{array}{l}\text { Presente } \\
\text { Ausente }\end{array}$ & $\begin{array}{r}15 \\
346\end{array}$ & $\begin{array}{r}4,2 \\
95,8\end{array}$ & $\begin{array}{r}4 \\
14\end{array}$ & $\begin{array}{r}26,7 \\
4,0\end{array}$ & $\begin{array}{r}11 \\
335\end{array}$ & $\begin{array}{l}73,3 \\
96,8\end{array}$ & $<0,001$ & $\begin{array}{c}6,68 \\
{[2,50 ; 17,85]}\end{array}$ \\
\hline $\begin{array}{l}\text { Ausência do eco glandular } \\
\text { endocervical }\end{array}$ & $\begin{array}{l}\text { Presente } \\
\text { Ausente }\end{array}$ & $\begin{array}{r}10 \\
351\end{array}$ & $\begin{array}{r}2,8 \\
97,2\end{array}$ & $\begin{array}{r}8 \\
10\end{array}$ & $\begin{array}{r}80,0 \\
2,8\end{array}$ & $\begin{array}{r}2 \\
341\end{array}$ & $\begin{array}{l}20,0 \\
97,2\end{array}$ & $<0,001$ & $\begin{array}{c}28,57 \\
{[14,40 ; 56,68]}\end{array}$ \\
\hline
\end{tabular}

IC, intervalo de confiança. 
colo uterino na mulher grávida sem antecedentes de PPT. O exame ultra-sonográfico morfológico do segundo trimestre é preconizado no período da $18^{\underline{a}}$ à $24^{\mathrm{a}}$ semana. Todavia, para avaliação minuciosa do coração fetal e de detalhes anatômicos, o período posterior à $21^{\mathrm{a}}$ semana mostrase mais adequado. Portanto, no período da 21aa à 24ํㅡㄹ semana, o médico pode verificar, de forma acurada, a anatomia fetal por meio de US morfológica e julgamos ser esta uma oportunidade de avaliar o colo através da US-TV.

Motivamo-nos a pesquisar marcadores morfológicos ultra-sonográficos do colo preditores de PPT. No presente estudo, adotamos a definição de afunilamento descrita por To et al., ou seja, a largura da abertura do orifício interno superior a 5 $\mathrm{mm}^{(\mathbf{2 1})}$. O sinal do afunilamento foi observado, assim, em 4,2\% da população estudada e em $22,2 \%$ das pacientes que evoluíram para PPT espontâneo. Tais índices encontram-se dentro dos limites observados entre os diversos serviços participantes do estudo multicêntrico americano coordenado por Iams et al. em $1996^{(\mathbf{1 5})}$.

Algumas pacientes apresentavam colo aparentemente muito longo com o sinal do afunilamento na região mais alta, em decorrência da contração do segmento inferior uterino. Após poucos minutos, observamos regressão do sinal e visualização adequada do canal com o relaxamento da região segmentar. Portanto, destacamos que o sinal do afunilamento pode mimetizar a dilatação do orifício cervical interno e, portanto, apresentar-se como falso indicador de prematuridade em situações de sobredistensão vesical, de contrações focais do segmento inferior ou de compressão imprópria do colo pelo transdutor ultra-sonográfico.

Atualmente, a importância da detecção do sinal do afunilamento no exame de USTV permanece controversa quanto ao seu valor preditor de prematuridade. Em nossas observações, destacamos na Tabela 1 que a distribuição da presença do sinal do afunilamento na predição do PPT nas diferentes idades gestacionais revela índices elevados de especificidade e VPN em todas as semanas gestacionais avaliadas. Os valores de sensibilidade foram mais elevados à medida que avaliamos idades mais precoces por ocasião do parto inferiores à $34^{\mathrm{a}}$, 33a e $32^{\mathrm{a}}$ semanas. Estes valores podem refletir uma tendência deste sinal morfológico de predizer o parto em idades gestacionais mais precoces e, portanto, com maior risco neonatal.

O estudo estatístico por meio da análise de regressão logística univariada mostrou que há associação estatisticamente significativa entre a presença do sinal do afunilamento e o PPT espontâneo (Tabela 3). Todavia, a análise de regressão logística multivariada não conseguiu mostrar significância estatística na associação com PPT ao comparar o sinal do afunilamento ao outro marcador (EGE ausente), em qualquer das idades gestacionais analisadas. Na Unifesp/EPM, o estudo com pacientes de alto risco para PPT não havia mostrado, em 1999, relação estatística entre o sinal do afunilamento e PPT ${ }^{(28)}$. Em trabalho publicado em 2001, To et al. verificaram que o afunilamento era mais freqüente em colos menores, porém este sinal morfológico não contribuiu para a predição do parto em idades gestacionais inferiores à 33a ${ }^{\text {a semana }}{ }^{(21)}$.

Ao iniciarmos este estudo, pudemos perceber o grande número de publicações que abordavam a biometria cervical durante a gestação. Um número menor, porém também considerável, de trabalhos científicos estudava o sinal do afunilamento. Entretanto, identificamos apenas três publicações de um grupo de Tóquio que enfocavam o epitélio endocervical durante o período gestacional ${ }^{(29-31)}$. Não observamos nenhuma publicação na literatura médica mundial que abordasse de forma concomitante a avaliação do sinal do afunilamento e o estudo do epitélio endocervical, e dispusemo-nos, pois, a iniciar este trabalho.

O termo "cervical gland area" foi utilizado pelo referido grupo japonês para descrever um novo sinal ultra-sonográfico a ser observado durante o período gestacional. A ausência das glândulas pode indicar maturação cervical e pode ser considerada como importante preditor de PPT espontâneo ${ }^{(\mathbf{3 0 , 3 1})}$. Neste estudo, modificamos o nome do sinal - originalmente descrito como "cervical gland area" - para eco glandular endocervical (EGE), pois julgamos o termo "eco" mais apropriado para descrever o achado quando comparado à palavra "área", a qual denota, na língua portuguesa, a medida de uma superfície. Utilizamos a expressão "eco" como um sinal ultra-sonográfico que indica uma faixa na imagem bidimensional, da mesma forma como é amplamente utilizado o termo "eco endometrial", o qual nomeia a região constituída pelo endométrio ${ }^{(35)}$.

Por tratar-se de estudo prospectivo na população geral, teoricamente de "baixo risco" para PPT, foi observada a ausência

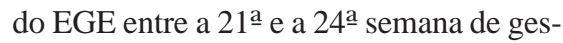
tação e a capacidade deste parâmetro de predizer o PPT espontâneo. Os estudos anteriores que avaliaram tal marcador ultra-sonográfico o fizeram em idades gestacionais distintas, constituindo, portanto, a primeira publicação nesta idade gestacional. Sekiya et al., em 1998, observaram pacientes em estudo longitudinal no segundo e no terceiro trimestres, entre a $16^{\mathrm{a}}$ e a $41^{\text {a }}$ semana de gestação ${ }^{(29)}$. Yoshimatsu et $a l$., em 2002, estudaram a quase totalidade das pacientes no terceiro trimestre ${ }^{(\mathbf{3 0})}$, e Fukami et al., em 2003, observaram pacientes entre a $16^{\underline{a}}$ e a $19^{\underline{a}} \operatorname{semana}^{(31)}$.

Observamos a ausência do EGE em $2,8 \%$ das pacientes estudadas entre a $21^{\text {a }}$ e 24ํㅗㅁ semana, enquanto Sekiya et al. encontraram a "cervical gland area" em todas as pacientes com idade gestacional inferior à $27^{\text {a }}$ semana $^{(29)}$. Fukami et al. verificaram a ausência do sinal em $0,4 \%$ da população avaliada entre a $16^{\underline{a}}$ e a $19^{\underline{a}}$ semana, portanto, em idades gestacionais mais precoces ${ }^{(\mathbf{3 1})}$ quando comparada à estudada por nós.

Questiona-se: a ausência da visualização do EGE apresentaria freqüência progressiva com o evoluir da gestação, como descrito inicialmente por Sekiya et al., porém com início mais precoce ${ }^{(29)}$ ? Estudos futuros longitudinais poderão esclarecer de forma mais acurada este padrão no decorrer da gestação. No que tange à ausência da EGE nas pacientes que tiveram PPT, nossos dados apontam freqüência maior quando comparada aos dados disponíveis na literatura. Verificamos EGE ausente em $44,4 \%$ das pacientes que evoluíram para PPT espontâneo, enquanto Fukami et al. observaram o fenômeno em apenas $8,3 \%$ das pacientes entre a $16^{-}$e a $19^{\underline{a}}$ semana de gestação que evoluíram para o PPT espontâneo ${ }^{(31)}$. 
A ausência do EGE na predição do PPT apresentou valores de sensibilidade mais elevados no grupo das pacientes com PPT em idades gestacionais mais precoces, como foi possível observar na Tabela 2. Acreditamos que uma elevação do tamanho amostral possa revelar de forma mais evidente esta tendência, particularmente nas pacientes com PPT em idades mais precoces, como mostrou a publicação de Fukami et al. ${ }^{(31)}$. Os autores destacaram a elevada sensibilidade (75\%) da ausência do EGE na predição do PPT espontâneo em idade gestacional inferior à $32^{2}$ semana e, entre a $32^{\underline{a}}$ e a $36^{-}$semana, sensibilidade de $2,3 \%{ }^{(\mathbf{3 1})}$.

Os índices de especificidade e VPN permaneceram elevados, em valores muito próximos a $100 \%$, em todas as idades gestacionais para predição de PPT espontâneo na ausência de EGE (Tabela 2). Isto está de acordo com os resultados de Fukami et $a l .{ }^{(31)}$. Tais dados indicam a reduzida probabilidade de PPT diante da visualização do EGE entre a $21^{\underline{a}}$ e a $24^{\underline{a}}$ semana.

A análise interpretativa do risco relativo obtido nas diferentes variáveis (Tabela 3) aponta a ausência do EGE como o sinal com risco relativo mais elevado $(28,57)$, seguido do sinal do afunilamento $(6,68)$. Estes dados indicam que o risco para PPT no grupo com ausência do EGE é 28 vezes maior que o risco para PPT no grupo com presença do EGE.

A ausência do EGE apresentou forte associação com o PPT espontâneo, estatisticamente significante em todas as idades gestacionais segundo a análise de regressão logística multivariada.

Os resultados desta pesquisa indicam que a ausência do EGE ao exame de US durante o segundo trimestre constitui o melhor preditor morfológico de PPT espontâneo quando comparado ao sinal do afunilamento, ao menos na população geral de gestações únicas. Entretanto, faz-se necessária a compreensão dos fatores que levam ao desaparecimento do EGE, de tal forma a entendê-lo como parâmetro para a predição do PPT.

O colo uterino é composto essencialmente por tecido muscular liso, colágeno e tecido conectivo. O colágeno da cérvice uterina constitui o principal componente estrutural mantenedor da integridade do colo no período anterior ao processo de parturição ${ }^{(36)}$. Durante a gestação, o colo uterino mantém consistência firme, forma alongada e o canal fechado, de tal modo a garantir a integridade das membranas e do concepto. No final da gravidez, apresenta um processo denominado "amadurecimento" ou "maturação", que o modifica de tal forma a torná-lo amolecido, encurtado e, finalmente, dilatado, para dar passagem ao feto $^{(37)}$.

O mecanismo de desencadeamento do parto na espécie humana ainda não se encontra completamente esclarecido ${ }^{(38)}$. As contrações uterinas, a ativação decidual e a competência cervical compreendem os componentes fundamentais do modelo contemporâneo da "síndrome do parto pré-termo espontâneo". Entretanto, a importância relativa e a interação entre esses fatores permanece apenas parcialmente conhecida $^{(38)}$.

Quando o processo de amadurecimento cervical ocorre precocemente na gestação, freqüentemente leva ao $\mathrm{PPT}^{(39)}$. Por conseguinte, uma estratégia lógica para predizer o PPT inicia-se com a identificação do amadurecimento cervical precoce. Mas qual seria o motivo do desaparecimento da imagem ultra-sonográfica correspondente às glândulas endocervicais (EGE) servir como marcador precoce do amadurecimento cervical? A mucosa endocervical é composta por epitélio colunar alto e contém glândulas amplas e muito ramificadas, que penetram no estroma cervical. De acordo com publicação de Fluhmann e Dickmann, da década de 1950, as glândulas endocervicais apresentam novas e mais pronunciadas invaginações durante o curso da gestação, definidas pelos autores como "tunnel clusters" ou cachos tuneli-

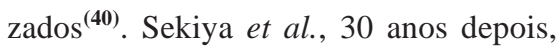
definiram a "cervical gland area" como sendo a região correspondente aos "tunnel clusters" da mucosa endocervical. Consequientemente, consideram que o desaparecimento deste sinal ultra-sonográfico seja decorrente do aumento do teor de água, de modificações bioquímicas do tecido conectivo $^{(\mathbf{2 9 )}}$.

Observamos, nas pacientes estudadas, que o EGE também apresenta variações da ecogenicidade nos diferentes exames. Habitualmente, a mucosa endocervical apre- senta-se hipoecóica quando o feixe acústico incide de forma perpendicular. Todavia, detectamos também mudanças do padrão ecogênico de acordo com o grau de inclinação do feixe, ou seja: quando o feixe ultra-sonográfico atinge a mucosa de forma mais oblíqua ou paralela, o eco tende a apresentar-se mais refringente. Neste trabalho, nas pacientes consideradas "EGE ausente", o eco não foi observado em nenhuma das inclinações.

Acreditamos que as modificações bioquímicas presentes no colo em processo de amadurecimento, secundárias ou não a processo infeccioso, promovam modificações arquiteturais importantes em decorrência da intensa colagenólise, o que poderia promover o colapso da estrutura das glândulas endocervicais e seu aplainamento, de forma concomitante ou precedente ao "apagamento" do colo. Outra hipótese, que poderia colaborar com o desaparecimento do EGE, consiste no acúmulo de água, o qual poderia levar à redução significativa da ecogenicidade do estroma e, portanto, dificultaria a visualização da faixa hipoecóica do $\mathrm{EGE}^{(\mathbf{3 1}, 36)}$. Estudos histológicos específicos do epitélio endocervical nas pacientes que apresentaram desaparecimento do EGE e/ou desenvolverem PPT espontâneo poderão auxiliar na elucidação dessas hipóteses.

Concluímos, diante do exposto, que a avaliação ecográfica do colo uterino na pesquisa de sinais de risco para PPT deve contemplar marcadores múltiplos, ou seja, biométricos e morfológicos, bem como a avaliação da significância de cada sinal encontrado. Este estudo apresenta limitações quanto ao tamanho amostral, porém revela uma tendência clara da marcante importância da ausência do EGE como indicador do risco para PPT, a ser confirmada em pesquisas multicêntricas futuras.

\section{REFERÊNCIAS}

1. Iams JD, Johnson FF, Creasy RK. Prevention of preterm birth. Clin Obstet Gynecol 1988;31:599615.

2. Guzman ER, Mellon C, Vintzileos AM, Ananth CV, Walters C, Gipson K. Longitudinal assessment of endocervical canal length between 15 and 24 weeks' gestation in women at risk for pregnancy loss or preterm birth. Obstet Gynecol 1998;92:317.

3. Guzman ER, Mellon R, Vintzileos AM, Ananth CV, Walters C, Gipson K. Relationship between endocervical canal length between 15-24 weeks ges- 
tation and obstetric history. J Matern Fetal Med 1998;7:269-72.

4. Goldenberg RL. The management of preterm labor. Obstet Gynecol 2002;100(5 Pt 1):1020-37.

5. Berkowitz GS, Papiernik E. Epidemiology of preterm birth. Epidemiol Rev 1993;15:414-43.

6. Bittar RE, Zugaib M. Assistência ao parto prematuro. Femina 2000;28:331-3.

7. Zemlyn S. The length of the uterine cervix and its significance. J Clin Ultrasound 1981;9:267-9.

8. Haram K, Mortensen JH, Wollen AL. Preterm delivery: an overview. Acta Obstet Gynecol Scand 2003;82:687-704.

9. Bittar RE, Yamasaki AA, Sasaki S, Galletta MA, Zugaib M. Determinação do risco para o parto prematuro através da detecção da fibronectina fetal na secreção cérvico-vaginal e da monitorização das contrações uterinas. Rev Bras Ginecol Obstet 1996;18:165-72.

10. Oliveira TA, Carvalho CMP, Souza E, et al. Avaliaçäo do risco de parto prematuro: teste de fibronectina fetal e medida do colo uterino. Rev Bras $\mathrm{Gi}_{-}$ necol Obstet 2000;22:633-9.

11. Iams JD, Goldenberg RL, Mercer BM, et al. The Preterm Prediction Study: recurrence risk of spontaneous preterm birth. National Institute of Child Health and Human Development Maternal-Fetal Medicine Units Network. Am J Obstet Gynecol 1998;178:1035-40.

12. Slattery MM, Morrison JJ. Preterm delivery. Lancet 2002;360(9344):1489-97.

13. Rozenberg P, Gillet A, Ville Y. Transvaginal sonographic examination of the cervix in asymptomatic pregnant women: review of the literature. Ultrasound Obstet Gynecol 2002;19:302-11.

14. Andersen HF, Nugent CE, Wanty SD, Hayashi RH. Prediction of risk for preterm delivery by ultrasonographic measurement of cervical length. Am J Obstet Gynecol 1990;163:859-67.

15. Iams JD, Goldenberg RL, Meis PJ, et al. The length of the cervix and the risk of spontaneous premature delivery. National Institute of Child Health and Human Development Maternal Fetal Medicine Unit Network. N Engl J Med 1996;334:567-72.

16. Heath VC, Southall TR, Souka AP, Elisseou A, Nicolaides KH. Cervical length at 23 weeks of gestation: prediction of spontaneous preterm delivery. Ultrasound Obstet Gynecol 1998;12:312-7.

17. Berghella V, Tolosa JE, Kuhlman K, Weiner S, Bolognese RJ, Wapner RJ. Cervical ultrasonogra- phy compared with manual examination as a predictor of preterm delivery. Am J Obstet Gynecol 1997;177:723-30.

18. Berghella V, Daly SF, Tolosa JE, et al. Prediction of preterm delivery with transvaginal ultrasonography of the cervix in patients with high-risk pregnancies: does cerclage prevent prematurity? Am J Obstet Gynecol 1999;181:809-15.

19. Hassan SS, Romero R, Berry SM, et al. Patients with an ultrasonographic cervical length $<$ or $=15$ $\mathrm{mm}$ have nearly a $50 \%$ risk of early spontaneous preterm delivery. Am J Obstet Gynecol 2000;182: 1458-67.

20. Guzman ER, Walters C, Ananth CV, et al. A comparison of sonographic cervical parameters in predicting spontaneous preterm birth in high-risk singleton gestations. Ultrasound Obstet Gynecol 2001;18:204-10.

21. To MS, Skentou C, Liao AW, Cacho A, Nicolaides KH. Cervical length and funneling at 23 weeks of gestation in the prediction of spontaneous early preterm delivery. Ultrasound Obstet Gynecol 2001;18:200-3.

22. Heath VC, Southall TR, Souka AP, Novakov A, Nicolaides KH. Cervical length at 23 weeks of gestation: relation to demographic characteristics and previous obstetric history. Ultrasound Obstet Gynecol 1998;12:304-11.

23. Yamasaki AA, Bittar RE, Fonseca ESB, Martinelli S, Sasaki S, Zugaib M. Prevenção de parto prematuro: emprego do toque vaginal e da ultra-sonografia transvaginal. Rev Bras Ginecol Obstet 1998;20: 350-6.

24. Berghella V, Kuhlman K, Weiner S, Teixeira L, Wapner RJ. Cervical funneling: sonographic criteria predictive of preterm delivery. Ultrasound Obstet Gynecol 1997;10:161-6.

25. Parulekar SG, Kiwi R. Dynamic incompetent cervix uteri. Sonographic observations. J Ultrasound Med 1988;7:481-5.

26. Brieger GM, Ning XH, Dawkins RR, et al. Transvaginal sonographic assessment of cervical dynamics during the third trimester of normal pregnancy. Acta Obstet Gynecol Scand 1997;76:118-22.

27. Owen J, Yost N, Berghella V, et al. Mid-trimester endovaginal sonography in women at high risk for spontaneous preterm birth. JAMA 2001;286: 1340-8.

28. Oliveira TA. Avaliação do risco de parto prematuro através do teste da fibronectina fetal e da me- dida do colo uterino pela ultra-sonografia transvaginal. (Tese de Doutorado). São Paulo, SP: Universidade Federal de São Paulo/Escola Paulista de Medicina, 1999.

29. Sekiya T, Ishihara K, Yoshimatsu K, Fukami T, Kikuchi S, Araki T. Detection rate of the cervical gland area during pregnancy by transvaginal sonography in the assessment of cervical maturation. Ultrasound Obstet Gynecol 1998;12:328-33.

30. Yoshimatsu K, Sekiya T, Ishihara K, Fukami T, Otabe T, Araki T. Detection of the cervical gland area in threatened preterm labor using transvaginal sonography in the assessment of cervical maturation and the outcome of pregnancy. Gynecol Obstet Invest 2002;53:149-56.

31. Fukami T, Ishihara K, Sekiya T, Araki T. Is transvaginal ultrasonography at mid-trimester useful for predicting early spontaneous preterm birth? J Nippon Med Sch 2003;70:135-40.

32. Hosmer DW, Lemesshow S. Applied logistic regression. New York, NY: John Wiley \& Sons, 1989.

33. Carvalho MH, Bittar RE, Brizot ML, Maganha PP, Borges da Fonseca ES, Zugaib M. Cervical length at 11-14 weeks' and 22-24 weeks' gestation evaluated by transvaginal sonography and gestational age at delivery. Ultrasound Obstet Gynecol 2003;21: $135-9$.

34. Carvalho MHB, Bittar RE, Gonzáles M, Brizot, ML, Zugaib M. Avaliação do risco para parto prematuro espontâneo pelo comprimento do colo uterino no primeiro e segundo trimestres da gravidez. Rev Bras Ginecol Obstet 2002;24:463-8.

35. Gonçalves WJ, Bortoletto CCR, Baracat EC, Lima GR. Ultra-sonografia e Doppler do endométrio. In: Pastore AR, ed. Ultra-sonografia em ginecologia e obstetrícia. 2 ${ }^{\underline{a}}$ ed. São Paulo, SP: Revinter, 2003: 665-79.

36. Danforth DN. The morphology of the human cervix. Clin Obstet Gynecol 1983;26:7-13.

37. Leppert PC. Cervical softening effacement and dilatation: a complex biochemical cascade. J Matern Fetal Med 1992;1:213-23.

38. Owen J, Iams JD, Hauth JC. Vaginal sonography and cervical incompetence. Am J Obstet Gynecol 2003; 188:586-96.

39. Winkler M. Role of cytokines and other inflammatory mediators. BJOG 2003;110 Suppl 20:118-23.

40. Fluhmann CF, Dickmann Z. The basic pattern of the glandular structures of the cervix uteri. Obstet Gynecol 1958;11:543-55. 